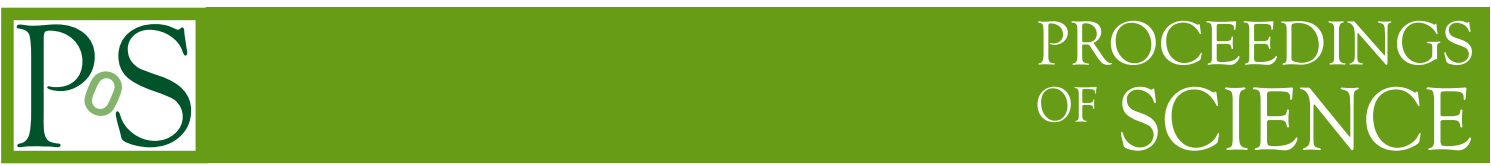

\title{
Methanol Multibeam Pulsar Survey
}

\author{
Jennifer O'Brien, ${ }^{1}$ S. Johnston, ${ }^{2}$ M. Kramer, ${ }^{1}$ A. G. Lyne, ${ }^{1}$ \\ M. Bailes, ${ }^{3}$ A. Possenti, ${ }^{4}$ M. Burgay, ${ }^{4}$ D. R. Lorimer, ${ }^{5}$ M. A. McLaughlin ${ }^{5}$ and \\ G. Hobbs ${ }^{2}$ \\ ${ }^{1}$ University of Manchester, Jodrell Bank Observatory, Macclesfield, Cheshire SK11 9DL, UK \\ ${ }^{2}$ Australia Telescope National Facility, CSIRO, P.O. Box 76, Epping NSW 1710, Australia \\ ${ }^{3}$ Swinburne Centre for Astrophysics and Supercomputing, Swinburne University of Technology, \\ Hawthorn, VIC 3122, Australia \\ ${ }^{4}$ INAF - Osservatorio Astronomica di Cagliari, 09012 Capoterra, Italy \\ ${ }^{5}$ Department of Physics, West Virginia University, Morgantown, WV 26506, USA \\ E-mail: Jennifer.0'Brien@manchester.ac.uk
}

\begin{abstract}
We present the initial results from a high-frequency survey of the Galactic plane for radio pulsars, using the 7-beam methanol multibeam receiver on the 64-m Parkes radio telescope. We describe briefly the survey system and some of the initial results. The survey has so far revealed one very interesting pulsar, which lies within an EGRET error box, meaning that it could be a radio-loud, gamma-ray pulsar. Another pulsar was discovered close to the Galactic centre.
\end{abstract}

From planets to dark energy: the modern radio universe

October 1-5 2007

University of Manchester, Manchester, UK 


\section{Introduction}

We report on the initial results of the Methanol Multibeam pulsar survey along the Galactic Plane. This survey utilises a seven beam methanol receiver, built in collaboration between Jodrell Bank Observatory and the Australian Telescope National Facility, operating at a wavelength of $5 \mathrm{~cm}$. The scientific aims of this survey are to discover young pulsars in the spiral arms, hidden at lower frequencies due to scattering, to improve our knowledge of the distribution of dispersing and scattering material, particularly in the inner Galaxy, to find rare objects e.g. a pulsar with a black hole companion and to survey the transient radio sky at high frequencies. The central observing frequency of $6306 \mathrm{MHz}$ is higher than usual for all-sky pulsar surveys, but is necessary for two reasons. The first is that pulse broadening due to interstellar scattering is quite bad at lower frequencies as the scattering time is proportional to $v^{-4}$. The main problem with scattering is that it cannot be corrected for post-detection. The second reason why a high frequency survey is a good option is that, at low Galactic latitudes, the background (synchrotron) radiation has an effective temperature which dominates the system temperature of the telescope at low frequencies, making it difficult to make clear observations. This is less of a problem at high frequencies.

\section{THE METHANOL MULTIBEAM SURVEY}

The survey of the Galactic plane for pulsars using the methanol multibeam receiver on the Parkes radio telescope commenced in February 2006. The survey observations are made in a 576$\mathrm{MHz}$ band centred on $6306 \mathrm{MHz}$.

The survey is split into two parts, a wide and a deep survey area. The wide survey covers an area of 55.2 square degrees, centred on the Galactic Centre, ranging from a longitude of $-60^{\circ}$ to $+30^{\circ}$ and a latitude between $\pm 0.25^{\circ}$. With 64 pointings per square degree that gives a total of 3360 pointings with an integration time per pointing of 1055 seconds. The deep survey concentrates on the Galactic Centre with 16 pointings and a longer integration time of 16800 seconds. The data are sampled at $0.125 \mathrm{~ms}$ and recorded on DLT-S4 tapes. The raw data is written directly to disk allowing approximately real time processing with a decimation of four. Full processing without decimation is performed at Jodrell Bank including a Fast Fourier Transform (FFT), a Fast Folding Algorithm and a single pulse search. The survey is estimated to takes 70 days to complete and we believe that the survey will give us an invaluable insight into high frequency pulsar surveys and the true nature of scattering material in the Galactic centre.

Survey observations are made on a hexagonal grid of pointings to give complete sky coverage, with adjacent beams overlapping at the half-power points. A total of 3376 pointings of the seven beams are required to cover the survey region. The survey is in its final stages with $94 \%$ of the observations completed to date. Of these completed observations, $71 \%$ have been processed using a FFT in the first pass through the data.

\section{Initial Results}

The wide survey has so far found one interesting pulsar. This recently confirmed pulsar, with a spin period of $50.05 \mathrm{~ms}$, lies within an EGRET error box, meaning that it could be a radio-loud, 

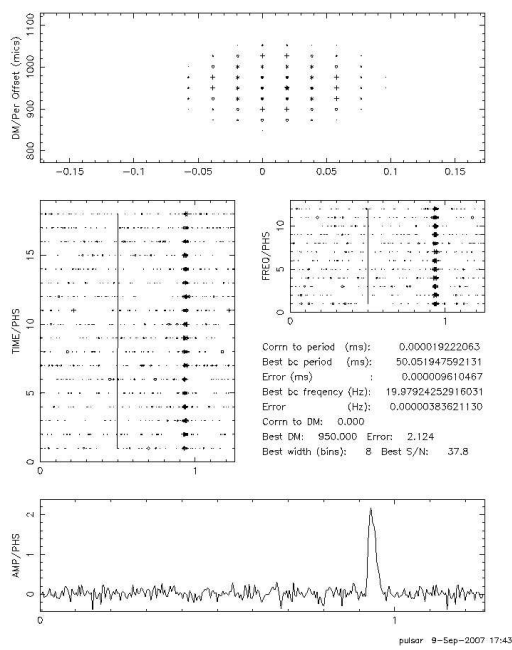

Figure 1: Confirmation plot of the pulsar taken at $\lambda 5 \mathrm{~cm}$ using the Parkes Telescope, ATNF. This pulsar is coincident with an EGRET error box.
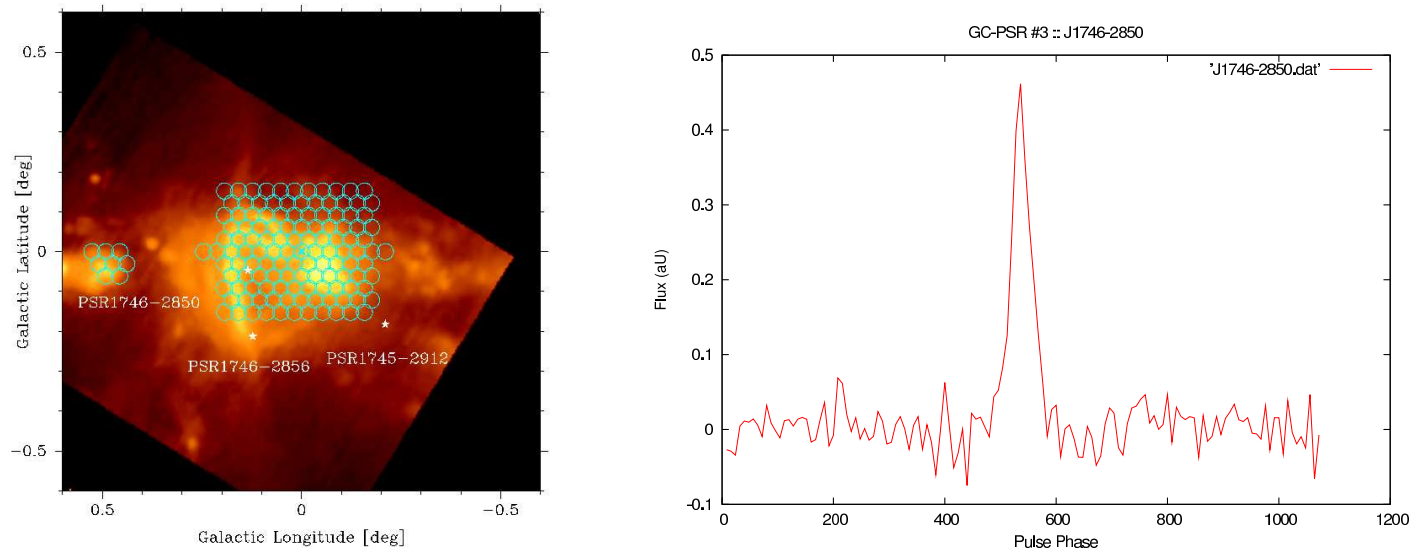

Figure 2: Left - Map of the Galactic Centre showing our newly discovered pulsar, J1746-2850, and two other nearby pulsars. The receiver beam positions from a previous Effelsberg survey are superimposed on top of the image. Right - Confirmation profile of the Galactic centre pulsar, taken with the Effelsberg telescope. Figures courtesy of Bernd Klein.

gamma-ray pulsar, (O'Brien et al. 2007, in prep.), see Figure 1. The deeper survey has found a Galactic Centre pulsar with spin period of 1170 ms, (Johnston et al. 2007, in prep), see Figure 2.

\section{References}

[1] A. G. Lyne and F. G. Smith, Pulsar Astronomy, Cambridge Astrophysics, 3rd Edition

[2] J. T. O'Brien et al. 2007, in preparation

[3] S. Johnston et el. 2007, in preparation 